\title{
Un recuento de los efectos que ha tenido el seguro obligatorio de automóviles en el régimen inglés de responsabilidad extracontractual
}

\author{
Daniel VÁsquez VeGA*
}

\section{Para citar este artículo/To cite this article}

Vásquez Vega, Daniel, Un recuento de los efectos que ha tenido el seguro obligatorio de automóviles en el régimen inglés de responsabilidad extracontractual, 43 RIS, 159-170 (2015). http://dx.doi.org/10.11144/Javeriana.ris43.reso doi:10.11144/Javeriana.ris43.reso

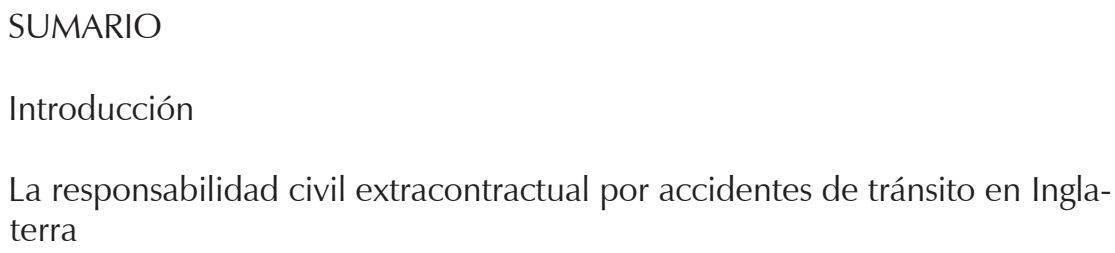
terra

El seguro obligatorio de automóviles y su impacto en el régimen de responsabilidad Inglés

Conclusión

Abogado de la Universidad CES (Col), Especialista en Derecho de Seguros de la Pontificia Universidad Javeriana (Col), LLM de UCL (RU), profesor de seguros en la maestría en Derecho y en la especialización de Responsabilidad Civil y Seguros de la Universidad Pontificia Bolivariana, en la especialización de Responsabilidad Civil y Seguros de la Universidad EAFIT, en la especialización de Derecho Comercial de la Universidad del Rosario dictada en Medellín en convenio con la Universidad CES y de diplomados y cursos en seguros de diferentes universidades. Abogado de la Dirección de Seguros de la Gerencia de Asuntos Legales de Suramericana. Contacto: dvasquezv@outlook.com. 


\title{
RESUMEN
}

Frecuentemente se afirma que entre la responsabilidad civil y los seguros hay una relación intrínseca y que las normas de estos terminan afectando a aquella. Para verificar si efectivamente existe dicha relación, este artículo analiza el caso puntual de los efectos que ha tenidos el seguro obligatorio de responsabilidad por accidentes de tránsito en el régimen inglés de responsabilidad extracontractual. Específicamente revisa la opinión manifestada por varios Lores de Justicia ingleses en sus fallos cuando analizan si a) ¿deben elevarse los estándares del deber de diligencia cuidado, porque al final la indemnización acordada será pagada por una compañía de seguros?; y b) ¿deben limitarse los perjuicios a indemnizar debido al impacto que la adjudicación de perjuicios elevados podrían tener sobre las primas de seguros y por lo tanto sobre la sociedad en general que las tiene que pagar?

Palabras clave: Seguros; seguro de responsabilidad; responsabilidad extracontractual; accidentes de tránsito; activismo judicial.

\begin{abstract}
It is often said that there is an intrinsic relationship that between tort law and insurance, and that the rules of the latter end up affecting those of the former. To see if there is indeed such a relationship, this article analyses the specific case of the effects that mandatory automobile insurance has had on English tort law. It specifically studies the views expressed by several Lord Justices in their judgments when they analyse whether a) the standards of the duty of care should be raised because in the end the damages awarded will be paid out by an insurance company?; and b) should the right or the amount of damages to be recovered be limited because of the impact that awarding high damages might have on insurance premiums and therefore on society in general who has to pay them?
\end{abstract}

Key words: Insurance; liability insurance; tort law; road traffic accidents; judicial activism. 


\section{INTRODUCCION}

Es común que se hable de la relación entre la responsabilidad civil y los seguros, en particular de la dependencia que en algunos casos existe entre los dos sistemas o del impacto e influencia que las normas de los seguros pueden tener en las de la responsabilidad a pesar de que aquellas no establezcan nada explícito sobre estas. ${ }^{1}$ Al respecto el profesor LAHNSTEIN indica que si bien "[a]ún no se reconocen de forma general las diversas interacciones entre la responsabilidad civil y el seguro", ${ }^{2}$ es claro que, por lo menos, la existencia de los seguros de responsabilidad ha conllevado a que: a) el legislador o los tribunales, consciente o inconscientemente, le asignen responsabilidad al causante del daño debido a la solvencia que le otorga el seguro; ${ }^{3}$ b) haya "menos escrúpulos a lo hora de establecer responsabilidad civil objetiva o de seguridad, y de ayudar a la víctima en la prueba de los hechos", lo cual afecta incluso los casos en los cuales no hay seguro; ${ }^{4}$ c) varíe la causalidad, esto es, dependiendo de las actividades aseguradas puede aumentar la probabilidad de que esa actividad sea identificada como la causa del daño; ${ }^{5}$ y d) se afecte la función preventiva del derecho de responsabilidad civil. ${ }^{6}$

1 Ver por ejemplo C Lahnstein, 'El seguro de responsabilidad civil y la responsabilidad social corporativa' en Responsabilidad civil, derecho de seguros y filosofía del derecho: Homenaje al profesor Javier Tamayo Jaramillo, Tomo II (Diké 2011) 999; R Lewis, 'Insurance and the tort system' (2005) Legal Studies 85; Juan Manuel Díaz-Granados, El seguro de responsabilidad (Universidad del Rosario 2012) 1-3.

$2 \quad$ Lahnstein (n 2) 1015.

3 Ibid 1002.

4 Ibid.

5 Ibid 1005.

6 Ibid 1011-1015. 
Este artículo pretende explorar cómo se ha evidenciado en la práctica este fenómeno, esto es, verificar cuál ha sido el papel que en algunos casos determinados ha desempeñado el seguro de responsabilidad en los regímenes de responsabilidad civil. Para ello se estudiará puntualmente el caso del derecho inglés en lo concerniente a las reglas de responsabilidad extracontractual por la conducción de vehículos automóviles.

Con este propósito en mente primero se describirá brevemente cómo está estructurada la responsabilidad civil extracontractual en Inglaterra, haciendo énfasis en el régimen de responsabilidad por la conducción de vehículos automóviles (SEcción I); luego se analizarán algunas decisiones proferidas por los tribunales ingleses después de la expedición de la Ley de Tráfico de 1930 (Road Traffic Act 1930) mediante la cual se creó el seguro obligatorio de automóviles que cubre a los conductores en contra del riesgo de incurrir en responsabilidad frente a un tercero como consecuencia de un accidente de tránsito (SECCIóN II); y por último, con base en los resultados arrojados por el análisis, se concluirá si, por lo menos para el caso inglés, es cierto que los seguros tienen algún impacto o influencia en los sistemas de responsabilidad a pesar de que las normas de aquellos no establezcan nada que explícitamente modifique las normas de estos (SECCIÓN III).

\section{SECCIÓN I - LA RESPONSABILIDAD CIVIL EXTRACONTRACTUAL POR ACCIDENTES DE TRÁNSITO EN INGLATERRA}

El sistema inglés de responsabilidad civil extracontractual, llamado law of tort, es bastante disímil de los modelos del derecho europeo continental, pues a diferencia de estos, aquel nunca eliminó las distinciones entre los variados delitos y cuasidelitos que dan origen a la responsabilidad. ${ }^{7}$ En este sentido, para pretender una indemnización es necesario encajar los hechos en uno de los delitos o cuasidelitos (torts) existentes y ello a su vez determinará las reglas y régimen de responsabilidad a aplicar. ${ }^{8}$ En el presente, los principales delitos o cuasidelitos que existen en el derecho inglés son el asalto (assault), la agresión (battery), la detención ilegal (false imprisonment), la negligencia (negligence) y la molestia (nuisance). Los tres primeros requieren de intención de causar daño, esto es de dolo; el cuarto, por regla general requiere de culpa y esta deberá ser probada por la víctima/demandante o se presumirá según el caso; el último hace referencia a los daños que se causan con el uso de la propiedad y, por lo menos en parte, comprende la responsabilidad por actividades peligrosas y por lo tanto, de acuerdo con establecido en el caso de Rylands v Fletcher, ${ }^{9}$ se enmarca en un régimen de responsabilidad objetiva. ${ }^{10}$

$7 \quad$ Esta diferencia, así como en general toda la estructura del sistema de responsabilidad extracontractual inglés, es consecuencia del desarrollo histórico del sistema, en particular de la necesidad de encajar las pretensiones en proformas creadas por la corona (writs). Mark Lunney y Ken Oliphant, Tort Law: Text and materials (Oxford University Press 2010) 1-17.

8 Ibid.

9 Este es un caso paradigmático mediante el cual se estableció en Inglaterra que para el caso de actividades peligrosas resultaba irrelevante el si había culpa o no del responsable.

Lunney (n 8) 640 y ss. 
En cuanto a la responsabilidad por accidentes de tránsito, antes de que se inventaran a finales del siglo XIX los primeros automóviles modernos, otros vehículos 'autopropulsado' que les precedieron habían sido considerados peligroso y por lo tanto, cuando los tribunales ingleses se enfrentaron a casos en los que estos vehículos causaban accidentalmente daños a terceros, fijaron un régimen de responsabilidad objetiva mediante la aplicación del principio establecido en Rylands v Fletcher. ${ }^{11}$ Sin embargo, cuando el primer caso de un accidente causado por un bus motorizado llegó a la Corte de Apelaciones en el caso de Wing v L.G.O.C., ${ }^{12}$ esta consideró que estos "nuevos" vehículos no eran ni lo suficientemente peligroso, ni una molestia como para caer dentro del régimen de responsabilidad objetiva establecido en Rylands v Fletcher. ${ }^{13}$ Este enfoque fue seguido en casos posteriores ${ }^{14}$ y fue extendido a los automóviles que hoy conocemos en el caso de Phillips v Britannia Hygienic Laundry. ${ }^{15}$ Desde entonces, el régimen de responsabilidad extracontractual establecido para los accidentes de tránsito no es uno de responsabilidad objetiva, sino uno basado en la culpa, lo que significa que si la víctima/demandante aspira a ser indemnizado por los daños y perjuicios derivados de un accidente de tránsito tiene que probar que ha habido una violación del deber de cuidado por parte del conductor del automóvil.

Las razones por las cuales se optó por un régimen de responsabilidad de este tipo (esto es, de culpa probada) han sido una fuente de debate. J. R. SPENCER, por ejemplo, ha dado a entender que, entre otras cosas, el hecho de que a principios del siglo XX el "automovilismo había sustituido al ciclismo como el deporte de la aristocracia progresista", a la cual pertenecía el poder judicial, hace que "no fuese sorprendente que los jueces hayan sido reacios a catalogar su nuevo hobby como una actividad peligrosa." ${ }^{\prime 16}$ Pero sea esto cierto o no, lo que no se puede negar es que desde la decisión de Wing v L.G.O.C. la responsabilidad extracontractual por accidentes de tránsito en Inglaterra se ha basado en la culpa y ni el Parlamento ni los tribunales han cambiado esa norma. ${ }^{17}$

Esto no quiere decir que no se hayan emitido leyes que regulen la circulación por carretera, sino simplemente que tales leyes no abordan la cuestión de la respon-

11 Ver por ejemplo el caso de Watkins v Reddin (1861) 2 F. \& F. 629 y el caso de Powel v Fall (1880) 5 Q.B.D. 597. En este último Baron Bramwell dijo que "Es justo y razonable que si una persona utiliza una máquina peligrosa deba pagar los daños que ella ocasione".

12 [1909] 2 K.B. 652.

13 J. R. SPENCER indica que las cortes dijeron que el principio de Rylands v Fletcher "podría en teoría aplicar a cosas peligrosas en la autopista, pero que nadie en sus correctos cabales podría decir que un bus motorizado era lo suficientemente peligroso como para caer dentro de la regla". Ver J. R. Spencer, "Motor-Cars and the Rule in Rylands v. Fletcher: a chapter of accidents in the history of law and motoring' (1983) CLJ 65, 72.

14 Parker v L.G.O.C. (1909) 73 J.P. 283.

15 [1923] 1 K.B. 539.

16 Spencer (n 14) 79.

17 Ha habido varios intentos en el Parlamento por modificar el régimen de responsabilidad por accidentes de tránsito, particularmente entre 1932 y 1934 y en 1975, pero en ambas ocasiones los proyectos se hundieron. 
sabilidad extracontractual directamente. Entre las leyes expedidas se destacan la Ley de Tráfico de 1930, la Ley de Tráfico de 1988 y el Código de Circulación. Como se verá a continuación, estas parecen haber tenido algún efecto en la forma en que los tribunales analizan el régimen de responsabilidad por accidentes de tránsito y, aunque todavía lo consideran como basado en la culpa, en algunos casos los jueces parecen soportarse en las disposiciones de dichas leyes para aumentar los estándares del deber de cuidado, haciendo prácticamente imposible que los demandados se puedan exonerar demostrando diligencia y cuidado, o para cambiar el monto de los perjuicios a indemnizar. Tal impacto y los debates generados se pueden observar, entre otros casos, en el efecto que el seguro obligatorio de automóviles, establecido y el artículo 30 de la Ley de Tráfico de 1930 y artículo 143 de la Ley de Tráfico de 1988, ha tenido sobre la responsabilidad extracontractual.

Es importante aclarar que, a diferencia de Colombia, en Inglaterra el seguro obligatorio de accidentes de tránsito es un seguro de responsabilidad civil. Con esto en mente se pasará a analizar el impacto o influencia que este ha tenido en el régimen de responsabilidad inglés.

\section{SECCIÓN II - EL SEGURO OBLIGATORIO DE AUTOMÓVILES Y SU IMPACTO EN EL RÉGIMEN DE RESPONSABILIDAD INGLÉS}

El efecto del seguro obligatorio de automóviles en el derecho inglés no ha sido uniforme porque los jueces han tenido diferentes puntos de vista sobre el peso que la existencia de este seguro debe tener en sus decisiones. Sus enfoques pueden examinarse desde dos perspectivas que guiarán el análisis de este artículo y que se enmarcan en las siguientes preguntas:

a) ¿Deben elevarse los estándares del deber de cuidado (facilitándole a las víctimas de un accidente de tránsito el obtener una indemnización), porque al final la indemnización acordada será pagada por una compañía de seguros?; y

b) ¿Deben limitarse los perjuicios a indemnizar debido al impacto que la adjudicación de perjuicios elevados podrían tener sobre las primas de seguros y por lo tanto sobre la sociedad en general que las tiene que pagar?

\section{Estándar del deber de cuidado}

En cuanto a la primera cuestión, es decir, si el estándar del deber de cuidado debe ser modificado, hay dos posiciones que se pueden observar en las decisiones de las Cortes. Por un lado, algunos jueces han considerado que, como desde la introducción del seguro obligatorio de automóviles la mayor parte de las indemnizaciones será pagada por las compañías de seguros, los estándares del deber deben ser variados hasta un punto que facilite la recuperación por parte de la víctima, sin que esto implique que 
el régimen de responsabilidad deje de ser uno de culpa. ${ }^{18}$

El principal proponente de este punto de vista era Lord DenNING M.R. quien en el caso de Nettleship v Weston ${ }^{19}$ dijo que:

"El alto nivel [de cuidado] así impuesto por los jueces es, creo, en gran parte el resultado de las políticas públicas derivadas de las leyes sobre el tránsito por carretera. El Parlamento exige que todo conductor esté asegurado contra los riesgos de terceros. La razón de esto, es que una persona lesionada por un automóvil no tenga que soportar la pérdida por su cuenta, sino que deba ser compensada con cargo al fondo de seguros. El fondo es más capaz de soportarla que él. Pero la persona lesionada sólo puede recuperar la pérdida si el conductor es responsable en derecho. Así que los jueces velan por que sea responsable, a menos que pueda demostrar diligencia y cuidado de un alto nivel."

Este punto de vista lo llevó incluso a declarar en la misma sentencia que

“estamos, en esta rama del derecho, alejándonos del concepto: 'Ninguna responsabilidad sin culpa.' Estamos empezando a aplicar la prueba: ‘¿En quién debería recaer el riesgo?' Moralmente el conductor no tiene la culpa; pero legalmente es responsable porque está asegurado y el riesgo debe recaer sobre él."

Su opinión era contundente. Si el efecto e impacto que el seguro obligatorio de automóviles ha tenido en la responsabilidad extracontractual tuviese que ser decidido con base en esta cita por sí sola, la respuesta sería evidente.

Sin embargo, la posición de DenNING ha sido controversial y no es compartida por todos los jueces ingleses. Otros han pensado que la decisión de los tribunales no debe variar por el hecho de que la compañía de seguros vaya a asumir los perjuicios concedidos. Aunque de forma menos vehemente, después del discurso de Lord DeNNING $^{20}$ en Nettleship v Weston, SaLmON L.J., otro de los jueces del tribunal, estuvo en desacuerdo con él diciendo que el grado de cuidado debido por un conductor hacia

18 Debido a que el precedente jurisprudencial solo puede ser cambiado por el Parlamento (o excepcionalmente por la Corte Suprema de Justicia, antes Cámara de los Lores) los jueces no tienen dentro de su arbitrio modificar el régimen de responsabilidad aplicable a una actividad en particular, es por esto que deben limitarse a modificar el estándar de culpa exigido.

19 [1971] 2 Q.B. 691.

20 Las sentencias de los tribunales colegiados ingleses están compuestas por un discurso de cada uno de los miembros del tribunal asignados al caso (normalmente son tres). En su discurso cada miembro da las razones que soportan lo que él considere es la decisión más adecuada al caso. La decisión adoptada es la de la mayoría independientemente de que no coincidan las razones dadas por los Lores de Justicia (Lords Justices). 
las personas en la carretera no es "afectado por si el conductor está asegurado o no". Lamentablemente, a pesar de que el tercer juez (MeGAw L.J.) estuvo del lado de Lord DENNING en el resultado, no mencionó el seguro como algo que debe ser tenido en cuenta o no por el juez para decidir el caso, sino que hizo hincapié en razones de políticas públicas para no crear varios estándares del deber de cuidado. Lo anterior implica que lo dicho por DeNNING no puede ser considerado más que obiter dicta.

No obstante, en adición a lo anterior, si bien en algunos otros casos no hay pronunciamientos explícitos de los jueces sobre el tema, es posible preguntarse si los tribunales hubiesen estado tan dispuestos a otorgar los perjuicios que otorgaron si no hubiese existido seguro alguno. ${ }^{21}$ Tal fue el caso de Henderson $v$ Jenkins ${ }^{22}$ en el que según J. R. SPENCER:

"[L]os tribunales hicieron magia negra con res ipsa loquitur y las presunciones de negligencia con el fin de afirmar que era negligente el dueño de un camión cuyos frenos habían fallado, a pesar de que había hecho todo lo que un propietario razonable habría hecho para mantenerlos, todo lo que el Ministerio de Transporte recomienda, y cuando los únicos pasos que podrían haber servido para detectar el defecto del freno fueron desalentados por el fabricante por motivos de seguridad." 23

La visión general de SPENCER sobre el asunto fue que:

"Una vez el seguro de responsabilidad civil para los automovilistas se hizo obligatorio en 1930, y fue la compañía de seguros del conductor la que pagó la indemnización en vez del propio conductor, incluso los partidarios del principio de culpabilidad ya no encontraban invariablemente injusto sostener responsable a un conductor a quien moralmente no podía imputársele culpa." ${ }^{24}$

Evidentemente, en cuanto a la primera cuestión, si bien el impacto que ha tenido la existencia de un seguro obligatorio de responsabilidad por accidentes de tránsito en el régimen de responsabilidad que aplica a estos casos no ha sido unánimemente reco-

21 Al analizar los efectos de los seguros en el sistema de responsabilidad civil extracontractual R. LewIS señala que "[e]n el pasado casi nunca se veía que los jueces examinaran o tan siquiera se percataran de la existencia de un seguro relacionado con la reclamación y aún ahora continúa siendo bastante inusual que lo hagan. En ocasiones han afirmado que los seguros no han tenido ningún efecto directo sobre ellos... [s] in embargo, su silencio general sobre estos asuntos no ha prevenido especulación sobre si los argumentos que hacen referencia al impacto de los seguros está teniendo un efecto no reconocido." Lewis (n 2) 94.

22 [1970] A.C. 282.

23 Spencer (n 14) 81.

24 ibid 81. 
nocido y aceptado por todos los jueces, explícita o implícitamente sí ha sido un factor determinante de las decisiones tomadas por ellos, por lo menos en algunos de los casos.

\section{Derecho a la indemnización de perjuicios y su importe}

La segunda pregunta, esto es, la relativa a si el derecho o el monto de la indemnización perjuicios que deben otorgar los jueces se debe limitar a causa del impacto que la adjudicación de altas indemnizaciones podría tener sobre las primas de seguros, fue planteada por primera vez por Diplock L.J. en el caso de Wise v Kaye. ${ }^{25}$ En este se estableció que una de las "principales consideraciones empíricas que determinará el importe máximo que se concederá para la peor lesión que puede ser prevista que ocurra" es la existencia de un "seguro de responsabilidad por daños por lesiones personales". Diplock señaló que debido a los seguros, la indemnización máxima que se fuera a conceder ya no tenía que ser la suma que los acusados eran capaces de pagar. Los seguros, dijo, "eliminan la carga inmediata de pagar daños y perjuicios a los acusados individuales y la extiende en última instancia, sobre el cuerpo general de los tomadores de seguros que pagan las primas." Hasta este punto su opinión se parece de alguna manera la de Lord DeNNING, pero Diplock L.J., reconociendo que el aumento de las indemnizaciones también aumenta el costo del seguro, "con la consiguiente dificultad para el público en general", dijo que el establecimiento de la máxima a indemnizar también debía tener esto en mente.

El enfoque de Diplock también se diferencia del de DenNing en que aquel consideraba que la existencia del seguro obligatorio no debía tenerse en cuenta en el caso a caso dependiendo de si había seguro o no, sino que sólo debía ser considerado en el establecimiento de los montos generales de la indemnización. ${ }^{26}$

El mismo problema con respecto a si el derecho a la indemnización debe ser limitado por el impacto que la concesión de indemnizaciones en algunas situaciones podría tener sobre las primas de seguros se presentó en el Tribunal de Apelación en el caso de McLoughlin v O’Brain ${ }^{27}$ y, luego, cuando se apeló, en la Cámara de los Lores. Ronald DWORKIn describió magistralmente el debate cuando usó el caso como un ejemplo en su libro el "Imperio de la justicia". ${ }^{28}$ Según DorwkIN, las políticas públicas

25 [1962] 1 Q.B. 638.

26 Después de explicar que el impacto que el aumento en las primas tendría en la sociedad en general debía ser tenido en cuenta al momento de fijar la cuantía de perjuicios a indemnizar, DıPLOCK indica que estas dos consideraciones están dirigidas exclusivamente a arribar al criterio que debe ser tenido en cuenta en la definición de todos los casos en general, pues ya en el caso puntual "estaría mal otorgar a un demandante en particular una indemnización mayor o menor de acuerdo con si el demandado es rico o pobre, asegurado o no asegurado."

27 [1981] Q.B. 599 (CA).

28 En el imperio de la justicia Dworkin utiliza McLoughlin v O'Brian como un ejemplo de un caso difícil, evidenciándose que tan complejo puede ser determinar el efecto que la regulación puede tener en los regímenes de responsabilidad. Ver Ronald Dworkin, Law's Empire (Hart Publishing 1998). 
invocadas por el Tribunal para no conceder perjuicios en casos como el citado eran, entre otras cosas, que "ello aumentaría el costo del seguro de responsabilidad civil, por lo que sería más costoso conducir y tal vez conllevaría a que algunas personas pobres no podrían conducir en absoluto." ${ }^{29}$ La Cámara de los Lores en cambio consideró que "un aumento en el precio del seguro de responsabilidad civil, sin importar qué tan inconveniente esto podría ser para la comunidad en su conjunto, no puede justificar la negativa a hacer cumplir los derechos y deberes individuales que antes han sido reconocidos y ejecutados." ${ }^{30}$ Este razonamiento llevó a la Cámara a permitir la apelación (esto es, a casar el fallo). ${ }^{31}$

La opinión de la Cámara de los Lores es entonces diferente de la de DıPLOCK L.J., porque él había reconocido que el efecto que aumentar el máximo de indemnización por daños podía tener en las primas de seguros era un factor a tener en cuenta. La Cámara de los Lores, sin embargo, reiteró su postura en el caso de Wells v Wells. ${ }^{32}$ En este Lord LloYD DE BerWICK, al analizar cuáles serían las consecuencias de su decisión, señaló que:

"A pesar de que esto se traducirá en una carga más pesada sobre estos acusados y, si el principio se aplica en todos los ámbitos, en el sector de los seguros en general, no puedo ver nada injusto... No hay duda de que las primas de seguros tendrán que aumentar con el fin de tener en cuenta la nueva tasa menor de descuento [de perjuicios]. Si esto es algo que el país puede permitirse o no, es un tema sobre el que no fueron abordados sus señorías. Así que no estamos en condiciones de formarnos una opinión en cuanto a las consecuencias más amplias."

Una opinión similar fue expresada por Lord STEYN en su discurso para el mismo caso.

En estas decisiones, tanto frente a la primera como a la segunda cuestión, se evidencia que a pesar de que las disposiciones de tránsito relacionadas con el seguro obligatorio han tenido un efecto sobre el régimen de responsabilidad, el efecto no ha sido uniforme pues para la misma cuestión ha habido tratamiento judicial mixto o, en muchos casos, ni siquiera se ha debatido explícitamente.

\section{Sección III - Conclusión}

Como se puede evidenciar a partir de los casos examinados, el efecto de los seguros sobre la responsabilidad civil extracontractual en Inglaterra es innegable. Sin embargo, el alcance de este efecto varía entre las diversas cuestiones que han sido discutidas por

29 ibid 27.

30 ibid 28.

31 McLoughlin v O'Brian [1983] 1 A.C. 410 (HL).

32 [1999] 1 A.C. 345 
los tribunales ingleses. El grado de impacto del seguro de obligatorio de automóviles es todavía un tema de debate o, al menos, de sospecha en los casos en que ni siquiera es mencionado por los jueces. De hecho, si bien el régimen de responsabilidad por culpa persiste a pesar de la introducción del seguro obligatorio de automóviles, los tribunales han admitido en muchos casos su importancia en la toma de sus decisiones. Por lo tanto, en general se puede afirmar que el seguro ha tenido un impacto visible en las disposiciones del derecho de responsabilidad civil en relación con los accidentes de tránsito, ya sea directa o indirectamente.

El análisis hecho por RICHARD LewIS en 'Insurance and the Tort System', aunque dirigido a los seguros en general, aplica a los seguros de automóviles en particular. Él indica que los seguros son "tenidos en cuenta, pero solo en algunos casos. Es difícil predecir cuándo y con qué efecto. Hay una incertidumbre considerable." Y termina señalando que "[t]odos los comentaristas por lo tanto concluyen que los seguros sin duda han tenido alguna influencia en la responsabilidad civil extracontractual; es solo la extensión de esa influencia lo que está en duda." ${ }^{33}$ A la misma conclusión se llega en este artículo.

Al comienzo de su escrito sobre el seguro de responsabilidad civil y la responsabilidad social corporativa el profesor Lahnstein indica que la respuesta a la cuestión sobre el papel que desempeña el seguro de responsabilidad en el desarrollo del derecho de la responsabilidad oscila entre el 'derecho en la teoría' y el 'derecho en la práctica'. ${ }^{34}$ Esperamos que este artículo sea un pequeño aporte para que la respuesta a la cuestión se aleje un poco del primero y se acerque un poco al segundo.

\section{BIBLIOGRAFÍA}

\section{Casos}

- $\quad$ Froom v Butcher [1976] Q.B. 286.

- Henderson v Jenkins [1970] A. C. 282.

- $\quad$ McLoughlin v O’Brain [1981] Q.B. 599 (CA).

- McLoughlin v O’Brian [1983] 1 A.C. 410 (HL).

- $\quad$ Nettleship v Weston [1971] 2 Q.B. 691.

- $\quad$ Parker v L.G.O.C. (1909) 73 JP 283.

- $\quad$ Phillips v Britannia Hygienic Laundry [1923] 1 KB 539.

33 Lewis (n 2) 85.

34 Lahnstein (n 2) 999. 
- $\quad$ Powel v Fall (1880) 5 Q.B.D. 597.

- $\quad$ Stanton v Collinson [2010] EWCA Civ 81.

- $\quad$ Watkins v Reddin (1861) 2 F. \& F. 629.

- Wells v Wells [1999] 1345 A.C.

- Wing v L.G.O.C. [1909] 2 K.B. 652.

- $\quad$ Wise v Kaye [1962] 1 Q.B. 638.

\section{Demás fuentes}

- Díaz-Granados J, El seguro de responsabilidad (Universidad del Rosario 2012).

- Dworkin R, Law's Empire (Hart Publishing, 1998).

- Lahnstein C, 'El seguro de responsabilidad civil y la responsabilidad social corporativa' en Responsabilidad civil, derecho de seguros y filosofía del derecho: Homenaje al profesor Javier Tamayo Jaramillo, Tomo II (Diké 2011) 999.

- Lewis R, 'Insurance and the tort system' (2005) Legal Studies 85.

- Lunney M y Oliphant K, Tort Law: text and materials (Oxford University Press 2010).

- Spencer JR, 'Motor-Cars and the Rule in Rylands v. Fletcher: a chapter of accidents in the history of law and motoring ' (1983) Cambridge Law Journal 65. 